BV acceptance of this article, the publisher or recipien acknowtedges the U.S. Government's right to retain a nonexclusive, royalty-free license in and to any copyright covering the article.

\title{
Baryon Distribution in Relativistic Heavy-Ion Collisions*
}

\author{
Cheuk-Yin Wong \\ Oak Ridge National Laboratory \\ Oak Ridge, TN 3783 ?
}

\author{
WINTER WORKSHOP ON NUCLEAR DYNAMICS III \\ Copper Mountain, Colorado \\ March 5-9, 1984
}

\section{DISCLAIMER}

This report was prepared as an account of work sponsored by an agency of the United States Government. Neither the United States Government nor any agency thereof, nor any of their employees, makes any warranty, express or implied, or assumes any legal liability or responsibility for the accuracy, completeness, or usefulness of any information, apparatus, product, or process disclosed, or represents that its use would not infringe privately owned rights. Reference herein to any specific commercial product, process, or service by trade name, trademark, manufacturer, or otherwise does not necessarily constitute or imply its endorsement, recommendation, or favoring by the United States Government or any agency thereof. The views and opinions of authors expressed herein do not necessarily state or reflect those of the United States Government or any agency thereof.

\footnotetext{
* Research sponsored by the Division of High Energy and Nuclear Physics, U.S. Department of Energy, under Contract W-7405-eng-26 with the Union Carbide Corporation.
} 
Baryon Distribution in Relativistic Heavy-Ion Collisions*

Cheuk-Yin Wong

Oak Ridge National Laboratory, Oak Ridge, TN 37831

Recently there is considerable interest in highly relativistic heavy-ion collisions ${ }^{1}$ which was suggested as a way to produce a phase transition from the ordinary confined matter to the unconfined quark-gluon plasma. As the fraction of baryons in the early universe was small, ${ }^{2}$ it seems desirable to design a heavy-ion collider such that when the energy density in the central rapidity is high enough for a phase transition, there is no net baryon density there.

Recent investigations reveal that the average downward shift of the projectile baryon rapidity is quite large. ${ }^{3}$ The formation of a pure quark-gluon plasma depends on the shape of the baryon momentum distributions. It is of interest to estimate the baryon momentum distribution.

We shall study the baryon distribution using the Glauber-type multiple collision model. ${ }^{4}$ In this model, a nucleon in one nucleus makes many inelastic collisions with nucleons in the other nucleus, the probability being given by the thickness function and the total nucleon-nucleon inelastic cross section. Each baryon-baryon collision results in a degradation of their energies and momenta in accordance with experimental nucleon-nucleon inelastic differential cross section data. This model is an extension of the model of Blankenbecler et al. 5 to include the effect of energy degradation. We shail focus our attentin $\overline{\text { on }}$ the longitudinal momentum distribution in terms of the Feynman scaling variable $x$.

We examine first the case of nucleon-nucleus collisions. The momentum distribution of the incident nucleon after $n$ inelastic collisions $D(n)\left(x_{n}\right)$ is related to that after $(n-1)$ inelastic collisions $D(n-1)\left(x_{n-1}\right)$ by

$$
D^{(n)}\left(x_{n}\right)=\int d x_{n-1} v^{(n-1)}\left(x_{n-1}\right) w\left(x_{n-1}, x_{n}\right) \text {. }
$$

where the function $w\left(x_{n-1}, x_{n}\right)$ is the probability for finding a "leading" baryon with $x_{n}$ after a baryon-baryon inelastic collision if the initial Feynman scaling variable is $x_{n-1}$. Experimental $p p \rightarrow p X$ data at high energies show a nearly flat ${ }^{6}$ and energy-independent ${ }^{7}$ differential cross section $d \sigma / d x$ as a function of $x$. Thus, the normalized probability distribution $w\left(x_{n-1}, x_{n}\right)$ can be approximated by

$$
w\left(x_{n-1}, x_{n}\right)=\theta\left(x_{n-1}-x_{n}\right) \theta\left(x_{n}-x_{L}\right) /\left(x_{n-1}-x_{L}\right) \text {, }
$$

where $x_{L}$ is the lower limit of $x$ in accordance with energy-momentum conservation. It is approximately the value of $x$ corresponding to the projectile taryon being stopped in the target frame or the rapidity variable $y$ being equal to the target rapidity. Initially, the momentum distribution is $D(0)(x)=\delta(x-1)$. The momentum distribution of the baryon after $n$ inelastic collisions is therefore 


$$
D^{(n)}(x)=\frac{1}{1-x_{L}} \frac{1}{(n-1) !}\left[-\ln \left(\frac{x-x_{L}}{1-x_{L}}\right)\right]^{n-1} \theta(1-x) \theta\left(x-x_{L}\right),
$$

which is normalized according to $\int D^{(n)}(x) d x=1$.

In the reaction $p A \rightarrow b X$ where $A$ is the target nucleus with mass number $A$ and $b$ is a baryon, the inelastic cross section $d \sigma \mathrm{PA} / \mathrm{dx}$ is

$$
\frac{d \sigma^{p A}}{d x}=\int d \vec{b} \sum_{n=1}^{A}\left(\begin{array}{l}
A \\
n
\end{array}\right) D^{(n)}(x)\left[T_{A}(b) \sigma_{i n}\right]^{n}\left[1-T_{A}(b) \sigma_{i n}\right]^{A-n} \text {. }
$$

where

$$
T_{A}\left(b_{A}\right)=\int d z_{A} \rho_{A}\left(b_{A}, z_{A}\right)
$$

and

$$
\int P_{A}(\vec{r}) d \vec{r}=1 .
$$

To calculate $d \sigma^{\mathrm{pA}} / \mathrm{dx}$ for the reaction $\mathrm{pA}+\mathrm{bX}$, we take a Fermi-type nuclear density distribution with a radius parameter $r_{0}=1.25 \mathrm{fm}$ and a diffuseness $a=0.523 \mathrm{fm}$. To compare with the experimental cross sections, we shall assume that in the range of interest, 0.3 $<x .<1$, the longitudinal and transverse degrees of freedom are factorizable. The theoretical results thus obtained give good agreement with experimental data $^{8}$ (Fig. 1).

We generalize the above results to the collision of a target nucleus $A$ with and a projectile nucleus with a mass number $B$. We neglect the collisions between projectile nucleons and the shift of the rapidities of the target nucleons. Within this approximation, all the projectile nucleons are alike and degrade in energy in the same manner. The projectile baryon distribution after the colitision is therefore

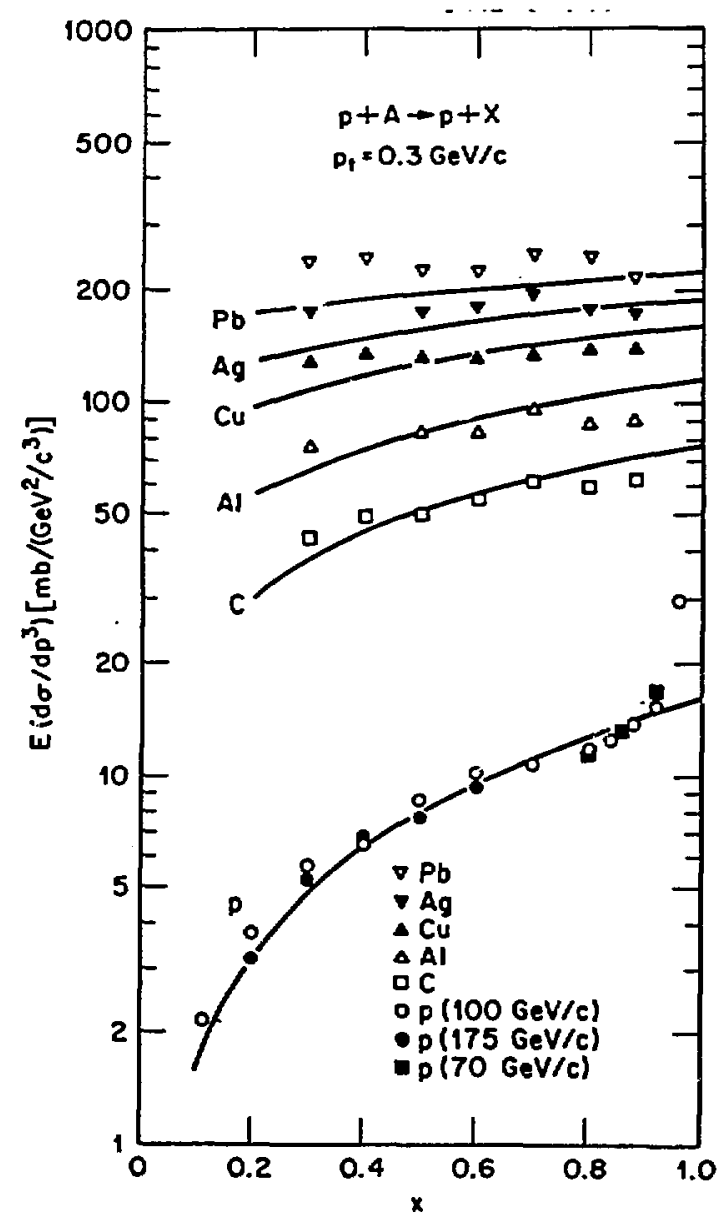




$$
\frac{d N}{d x}(x)=B \int d \vec{b}_{B}^{+} T_{B}\left(\vec{b}_{B}\right) \underset{n=0}{A}\left(\begin{array}{l}
A \\
n
\end{array}\right) D^{(n)}(x)\left[T_{A}\left(\vec{b}+\vec{b}_{B}^{+}\right) \sigma_{i n}\right]^{n}\left[1-T_{A}\left(\vec{b}^{+}+b_{B}\right) \sigma_{i n}\right]^{A-n}
$$

We calculate the part of projectile baryons which suffer at least one inelastic collision, $d N_{I N}^{B} / d y$, for the collisions of equal nuclei at two different bombarding energies. After taking into account the Fermi motion, the results are shown in Fig. 2 where we plot the quantity $\left(d N_{I N}^{B} / d y\right) / \pi R_{A}^{2}$ as a function of $y-y_{B}$ with $R_{A}$ the radius of the target nucleus and $y_{B}$ the beam rapidity. Figure $2(a)$ is for a bombarding energy of $\sqrt{5} / A=30 \mathrm{GeV}$ per nucleon and $F i g .2(b)$ is for $\sqrt{\mathrm{s}} / A=100 \mathrm{GeV}$ per nucleon. This quantity gives the proper baryon density in the central rapidity region at the proper time $t_{0}$ of $1 \mathrm{fm} / c$ when the produced mesons and other particles begin to emerge. 1 As the mass of the colliding nuclei increases, the peak of the projectile baryon distribution becomes wider and moves to a lower rapidity. The distribution is not symmetrical about the peak. It extends well into the target rapidity region.

Df particular interest is the baryon spatial density in the central rapidity region. It has contributions from both the projectile nucleus and the target nucleus. For $/ \mathrm{s} / \mathrm{A}=30$ GeV per nucleon, the midrapidity point is at $y-y_{B}=$ -4.16. The total baryon density there at $t_{0}=1 \mathrm{fm} / \mathrm{c}$ is 0.04 baryon $/ \mathrm{fm}^{3}$ for $\mathrm{C}+0,0.14$ baryon/ $/ \mathrm{fm}^{3}$ for $\mathrm{Cu}+\mathrm{Cu}$, and 0.28 baryon $/ \mathrm{fm}^{3}$ for $\mathrm{Pb}+\mathrm{Pb}$. For the higher energy $\checkmark / s / A=100 \mathrm{GeV}$ per nucleon, the mid-rapidity point is at $y-y_{B}=-5.36$. The total baryon density there at $t_{0}=1 \mathrm{fm} / \mathrm{c}$ is 0.02 baryon $/ \mathrm{fm}^{3}$ for $0+0,0.08$ baryon $/ \mathrm{fm}^{3}$ for $\mathrm{Cu}+\mathrm{Cu}$, and 0.21 baryon $/ \mathrm{fm}^{3}$ for $\mathrm{Pb}+\mathrm{Pb}$. One can compare these densities with the energy densities of the produced mesons in this mid-rapidity region. ${ }^{9}$ One finds that the baryon energy density is about $6 \%$ of the total energy density for $\checkmark s / A=30 \mathrm{GeV}$ per nucleon, and is about 2 to $3 \%$ for $\sqrt{ } / A=$ $100 \mathrm{GeV}$ per nucleon.

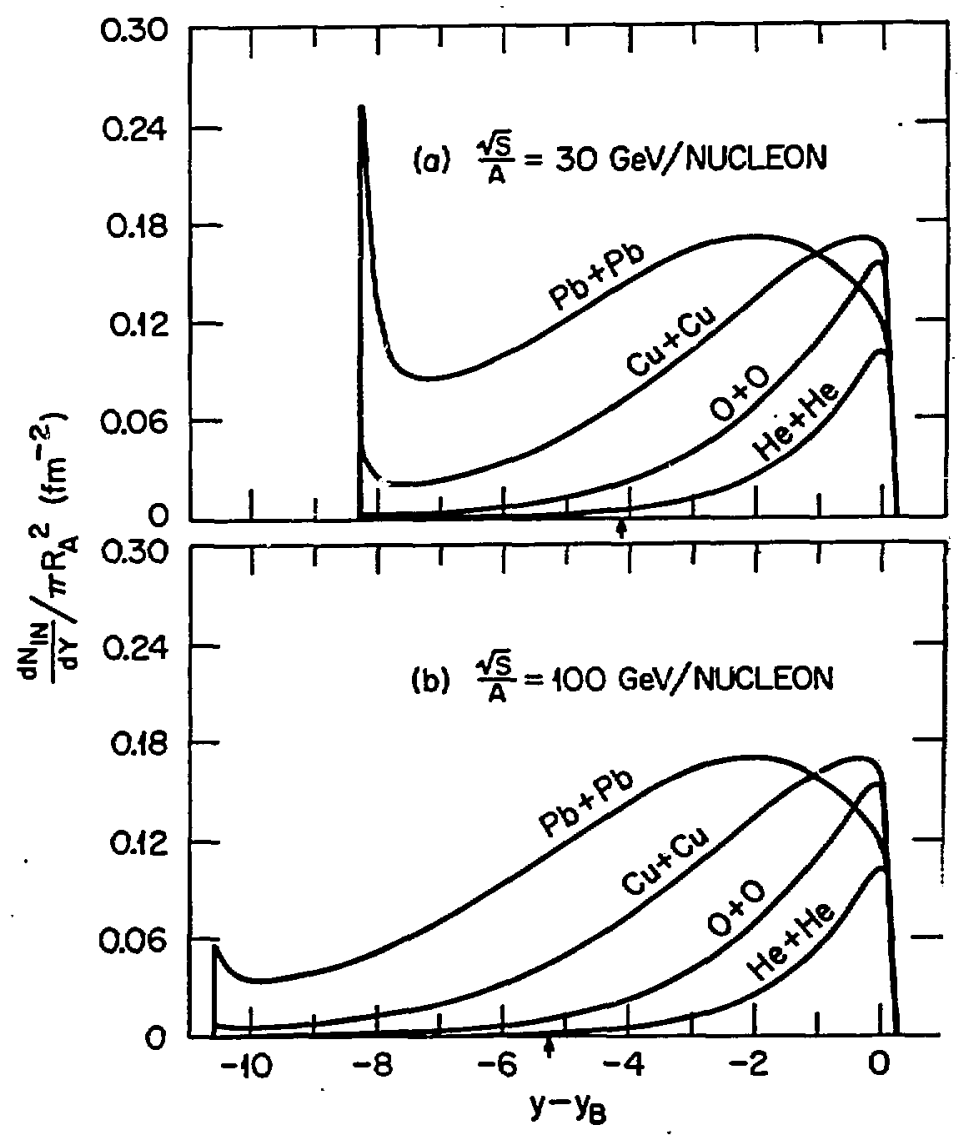

Figure 2 
Our result indicates that in the eniergy range of $\sqrt{\mathrm{s}} / \mathrm{A} \sim 10-100 \mathrm{GeV}$, it is difficult to form a pure quark-gluon plasma in the mid-rapidity region without a baryon impurity of a few percent (in energy density). Increasing the collider energy brings some improvement to the degree of baryon impurity. However, by nature of the skew distributions due to multiple inelastic collisions, some baryon impurity remains.

\section{REFERENCES}

* Research sponsored by the Division of High Energy and Nuclear Physics, U.S. Department of Energy, under contract W-7405-eng-26 with the Union Carbide Corporation.

1. J. D. Bjorken, Phys. Rev. D 27, 140 (1983) and references cited therein.

2. D. Schramm, Proceedings of the Third International Conference on U1traRelativistic Nucleus-Nucleus Collisions, Brookhaven National Laboratory, September 1983 (Fermilab Report Conf-83/92-AST).

3. W. Busza and A. S. Goldhaber, Proceedings of the Third International Conference on UItra-Relativistic Nucleus-Nucleus Collisions, Brookhaven National Laboratory, September 1983 (SUNY Institute of Theoretical Physics preprint ITP-SB-82, 1983).

4. R. J. Glauber, Lectures in Theoretical Physics, ed. by W. E. Brittin and L. G. Dunham, VoT. 1, p. 315, Interscience, New York (1959).

5. R. Blankenbecler, A. Capella, J. Tran Thanh Van, C. Pajares, and A. V. Rama110, Phys. Lett. 107B, 106 (1981).

6. A. E. Brenner et al., Phys. Rev. D 26, 1497 (1982).

7. F. E. Taylor et aT., Phys. Rev. D 14, 1217 (1976).

8. D. S. Barton et aT., Phys. Rev. D 2T, 2580 (1983).

9. C. Y. Wong, ORNL preprint, submitted to Physical Review D. 